\title{
A Guide for Mitochondrial DNA Analysis in Non-Human Forensic Investigations
}

\author{
Filipe Pereira ${ }^{1, *}$, João Carneiro ${ }^{1}$ and Barbara van Asch $^{1,2}$ \\ ${ }^{I}$ Instituto de Patologia e Imunologia Molecular da Universidade do Porto (IPATIMUP), Portugal \\ ${ }^{2}$ Faculdade de Ciências da Universidade do Porto, Portugal
}

\begin{abstract}
In recent years, mitochondrial DNA (mtDNA) typing has become established as a powerful tool for forensic investigations. The sequencing of mtDNA is now a standard laboratory procedure for the examination of degraded casework samples of human origin, with unique advantages over nuclear DNA profiling systems. Nonetheless, the use of mtDNA in non-human forensic science has only recently gained attention, with applications in the profiling of 'silent witnesses' of crimes, traceability of food products and control of illegal trade of endangered species or drugs, among others. This review briefly describes some important features of mtDNA with relevance for forensic testing in different taxonomic groups and suggests appropriate laboratorial and analytic procedures to avoid erroneous interpretations. It is imperative to merge a solid knowledge of mitochondrial genetics with rigorous methods of error prevention in order to permit the full approval of non-human mtDNA profiling in routine forensic work.
\end{abstract}

Keywords: Mitochondrial DNA, non-human forensics, laboratorial procedures, data interpretation.

\section{SPECIAL FEATURES OF MITOCHONDRIAL DNA}

Mitochondria are cellular organelles that provide most of the energy that organisms need to live. All eukaryotic cells are believed to contain at least some mitochondria or a related organelle that evolved from free-living alphaproteobacteria [1]. These semi-autonomous organelles possess their own genetic material (mitochondrial DNA or mtDNA), encoding essential proteins of the oxidative phosphorylation system and the necessary RNA machinery (rRNAs and tRNAs) for the translation of mtDNA transcripts $[2,3]$. Some of the protein components of the mitochondrial ribosome are also encoded in the mtDNA of plants and protists. Since almost all eukaryotic species have mitochondria, forensic investigations can benefit from the analysis of mtDNA in a wide range of species.

The well-conserved function of mtDNA contrasts with its remarkable genetic variation in terms of conformation, size, gene content and arrangement, as observed across different eukaryotic species [4]. The mitochondrial genome typically consists of a single circular molecule, but many organelle genomes also exist as linear head-to-tail concatamers (e.g., in some ciliates, apicomplexa, fungi, green algae and cnidarian species) or as minicircular chromosomes (for instance, in the sucking louse, Pediculus humanus [5]). In terms of genome size, the two main multicellular groups of eukaryotes (animals and land plants) have evolved in radically different ways: plant mtDNA ranges in size from approximately 180 to $700 \mathrm{kbp}$ (reaching 773,279 bp in Vitis vinifera [6]; Fig. 1), whereas animal mtDNA generally falls in the range of

*Address correspondence to this author at the, IPATIMUP, Rua Dr. Roberto Frias s/n, 4200-465 Porto, Portugal; Tel: +351 22 5570700; Fax: +351 22 5570799; E-mail: fpereira@ipatimup.pt
14 to $20 \mathrm{kbp}$ with an extremely low proportion of noncoding DNA [7] (see Fig. 2 for an example). The largest expansions of mitochondrial genome size in plants, algae, fungi and some protists are only weakly associated with an increase in gene number and result mostly from the proliferation of introns, pseudogenes, transposable elements or large repetitive elements. The smallest mitochondrial genomes sequenced to date are those from malaria parasites, at less than $6 \mathrm{kbp}$ (Plasmodium and related genera; Fig. 3).

The main advantage of mtDNA in forensic investigations lies in its high number of copies per cell: usually there are several mtDNA molecules per mitochondrion and thousands of mitochondria in each cell. Together with the protection offered by a two-walled organelle, this feature increases the probability of obtaining results from degraded/low-copy DNA samples for which nuclear DNA fails to give a result (e.g., bones, hair, faeces, processed wood, food products, etc). The circular shape of most mtDNA molecules also prevents its degradation by exonucleases, a characteristic that increases the possibility of obtaining results from critical samples.

The polyploid nature of the mitochondrial genome has an important consequence in forensic mtDNA profiling: there is the possibility of two or more different mtDNA sequences existing in the same cell or organism (a phenomenon known as heteroplasmy). Unlike nuclear DNA, the replication of mtDNA in somatic cells occurs independently of the cell cycle by a process of relaxed replication [8]. Thus, all available evidence indicates that in multicellular species, somatic mutations in mtDNA accumulate throughout the lifetime of an individual from clonal expansion of individual mitochondrial genomes with either point mutations or insertions/ deletions (indels) $[2,9,10]$. This means that different tissues 


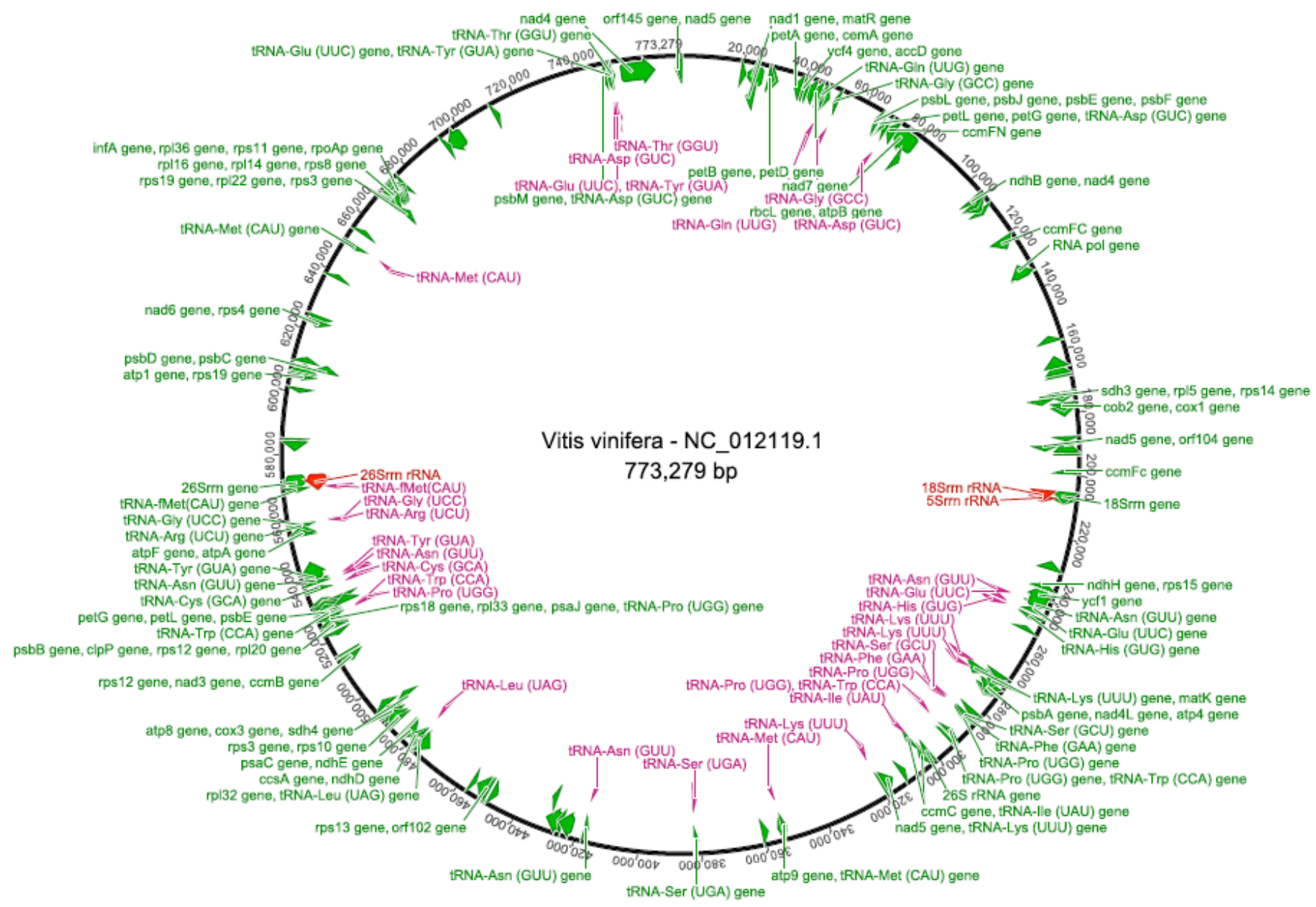

Fig. (1). Mitochondrial genome of Vitis vinifera. Image generated by Geneious software (http://www.geneious.com).

from the same individual may exhibit different mtDNA haplotypes and that their frequency can change with age. In practical terms, the frequency of heteroplasmy is usually very low because acquired mutations do not necessarily occur at the same site and are distributed in a highly heterogeneous manner among different tissues and within the same tissue, meaning that they remain largely undetected by standard sequencing techniques $(20 \%$ frequency is the threshold of detection for most techniques) [11-13].

In animals, mtDNA is typically transmitted to offspring exclusively by one parent (strict uniparental inheritance), although independent transmission of paternal mtDNA occurs in some bivalve species (doubly uniparental inheritance) [9, 14]. The occurrence of biparental inheritance (e.g., a small number of paternal mitochondria succeed in persisting in the zygote) has been documented in very few cases and usually in patients with mitochondrial disorders [15]. In plants and algae, mitochondria may be inherited from different sexes or mating types but are usually transmitted only by one parent. The simple pattern of inheritance can be useful to support or refute the identity of putative samples by comparison with reference samples from known relatives. Even for species with high mitochondrial mutation rates, mtDNA types are consistently transmitted across many generations. Thus, the presence of identical sequences in a large number of related individuals can be an important practical advantage when establishing the origin of a biological specimen, since multiple reference samples may be available for comparison (distant relatives can be used) [11-13].

The various arrangements of genes observed in the mtDNA of several taxonomic groups [4] suggest that intramolecular and intermolecular recombination occurs at the cellular level. However, there is little evidence that it has a significant impact at the population level (inter-lineage recombination) [9]. The fact is that organelle genomes are usually inherited uniparentally and rarely have an opportunity to recombine. Even if they are inherited biparentally, the organelles from the two parents do not necessarily fuse and recombine their genomes. In humans, the debate on mtDNA paternal inheritance and recombination is intense, but there is no clear case of recombination, and just a few examples of paternal mtDNA transmission have been reported [15-17]. The lack of recombination between lineages in many species means that, in those cases, individuals have a single haplotype (disregarding heteroplasmy) and that the interpretation of genetic analysis can be relatively straightforward. New lineages (or haplotypes) are generated solely by the sequential accumulation of mutations, and their frequencies are governed primarily by migration and genetic drift in populations. All forensic interpretations must take into account that all descendents of the same female (or male, if mtDNA is paternally transmitted) share the same haplotype. Exceptions to this rule could result from the accumulation of new somatic mutations in the descendant (usually causing hetero- 


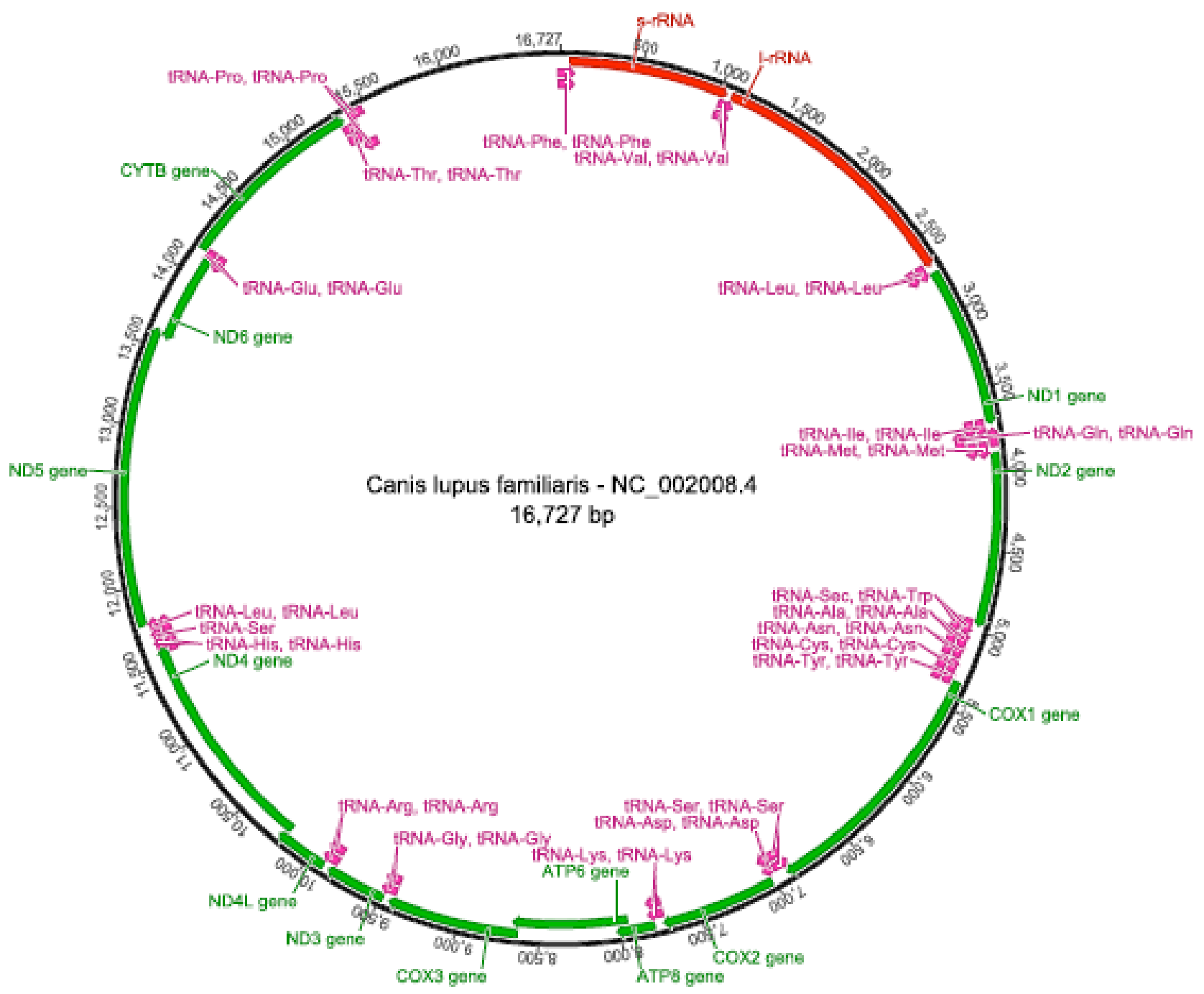

Fig. (2). Mitochondrial genome of Canis lupus familiaris. Image generated by Geneious software (http://www.geneious.com).

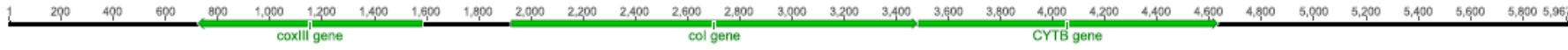

Fig. (3). Mitochondrial genome of Plasmodium falciparum. Image generated by Geneious software (http://www.geneious.com).

plasmy) or from differential segregation of pre-existing heteroplasmic variants in the germline of the progenitor that transmits the mitochondria [18]. As it has been shown in mammals, a progenitor with heteroplasmic germline cells may transmit widely varying levels of different mtDNAs to their offspring due to a bottleneck phenomenon during embryogenesis [19]. For this reason, maternal relatives (or paternal, in some species) can differ from one another by some mutations, particularly in hypervariable regions, which should be taken into account when interpreting the results in order to avoid false exclusions in identity testing.

Another important advantage of using mtDNA for forensic analysis is the large number of sequences available in public databases from population genetic, phylogeographical and phylogenetic studies [11, 13] (Box 1). These data can help in the construction of population databases, either to estimate the significance of a mtDNA haplotype in forensic casework $[13,20]$ or to use as a tool for species identification [21-24].

\section{DELINEATING A STRATEGY FOR USING mtDNA IN NON-HUMAN FORENSIC INVESTIGATIONS}

The success of a mtDNA-based forensic test largely depends on the choice of the appropriate genomic region to examine. Usually, the mtDNA regions of interest for discrimination of closely related lineages are those with a rapid rate of sequence evolution, as a result of a high mutation rate and/or low functional constraint. A more conserved region should be selected to discriminate individuals belonging to lineages with a high phylogenetic divergence, such as those from different species. In general, the major taxonomic groups have considerably different mitochondrial mutation rates. For instance, mutation rates estimated from phylogenetic comparisons under the assumption of neutral silent sites revealed that plants have a 100 -fold lower mutation rate of mtDNA $\left(0.34 \times 10^{-9}\right.$ base substitutions per site per year) than do animals (from $15.43 \times 10^{-9}$ in reptiles/amphibians to $33.88 \times 10^{-9}$ in mammals) $[7,25]$. This means that animals have a mtDNA mutation rate that is 10 to 25 times higher 


\begin{tabular}{|llrlr|}
\hline Box 1. Main taxonomic groups with complete mitochondrial genomes available \\
\hline Taxonomic group & Species & $n$ & \multicolumn{2}{c|}{ Genome size } \\
& & 32 & \multicolumn{1}{c|}{ smallest } & largest \\
Viridiplantae (green plants) & Maize, common tobacco, sorghum & 12,998 & 773,279 \\
Stramenopiles (heterokonts) & Brown algae, potato late blight agent & 15 & 27,719 & 58,507 \\
Fungi & Baker's yeast, oyster mushroom & 58 & 18,844 & 109,103 \\
Alveolata (alveolates) & Malaria parasite & 26 & 5,893 & 47,691 \\
Porifera (sponges) & Giant barrel sponge, marine sponge & 21 & 16,755 & 26,300 \\
Cnidaria (cnidarians) & Sea anemone, stony coral & 34 & 7,686 & 21,374 \\
Echinodermata (echinoderms) & Common urchin, crinoid, starfish & 20 & 14,837 & 17,538 \\
Platyhelminthes (flatworms) & Tapeworm, human blood fluke & 29 & 13,503 & 16,901 \\
Nematoda (roundworms) & Pig roundworm, human hookworm & 29 & 12,626 & 26,194 \\
Arthropoda (arthropods) & Fruit fly, house centipede, mantis shrimp & 234 & 12,984 & 24,961 \\
Mollusca (mollusks) & Common octopus, giant squid, sea scallop & 53 & 13,670 & 32,115 \\
Actinopterygii (ray-finned fishes) Zebrafish, Atlantic salmon, Pacific halibut & 539 & 15,564 & 19,527 \\
Amphibia (amphibians) & African clawed frog, redwood salamander & 84 & 15,897 & 22,874 \\
Lepidosauria (lepidosaurs) & Common chameleon, crocodile lizard, ball python & 70 & 15,181 & 25,972 \\
Testudines (turtles) & Green seaturtle, four eyed turtle & 29 & 16,455 & 19,438 \\
Aves (birds) & Domestic goose, peregrine falcon, ostrich & 98 & 15,574 & 18,967 \\
Metatheria (marsupials) & Common wombat, koala, Tasmanian wolf & 31 & 15,353 & 17,734 \\
Eutheria (placentals) & Dog, giant panda, polar bear, orangutan, whale & 238 & 15,289 & 17,411 \\
& & & & \\
& & & &
\end{tabular}

than in the nuclear genome, while in plants silent-site substitution rates are much lower in organelle sequences compared to nuclear sequences. It should also be made clear that these are mean values and that mutation rates vary along the mitochondrial genome, as some base positions appear to be very stable while others are highly mutable.

The mtDNA of plants exhibits a significant structural plasticity that contrasts with its low mutability: large genomic rearrangements, genes interrupted by introns, high recombinatorial activity, the incorporation of foreign DNA from the nuclear and chloroplast genomes and recurrent transfer of genes to the nucleus, among other features [26, 27]. These frequent genomic alterations can be used to trace specific lineages, even within a species (e.g., maize [28] and rye [29]). However, the technical difficulties in detecting most of these events still impedes the regular use of mtDNA, and a better comprehension of intraspecific diversity patterns is essential to the development of more reliable typing strategies. In animals, mtDNA has a very simple genetic structure without features such as introns, pseudogenes, transposable elements or large repetitive elements (with a few exceptions [30]). Only in very rare examples can the use of genomic rearrangements be useful for species identification procedures in animals. The immense structural and genetic diversity found in the mtDNA of the myriad groups of fungi, protists and green algae $[1,4]$ makes it difficult to delineate a universal mtDNA typing strategy. A detailed examination of all available data for the target group under investigation is highly recommended.

The first aspect to be considered for the development of mtDNA forensic analysis is the availability of a high-quality, complete mitochondrial sequence for the target species. The list of reference mtDNA genomes can be found in the NCBI Organelle Genome Resources (see Box 2 for web link). Additional information can be retrieved from diverse databases of mitochondrial genome information (Box 2). A standardized nomenclature and numbering of positions based on the earliest reported complete mtDNA sequence (if available) should be used for descriptions of polymorphisms and PCR primers, thus allowing for inter-laboratory comparisons and data reporting. When describing a mtDNA profile, only differences between the sample sequence and the reference sequence should be noted; all other positions in the sequence are understood to be the same as in the reference. In mammals, the two mtDNA strands are historically designated as heavy (H) and light (L) strands based on their different buoyant densities in a caesium chloride gradient, which results from a strand bias in base composition (the $\mathrm{H}$-strand is guanine-rich, whereas the L-strand is guanine-poor). The 5'3 ' L-strand is usually used as the reference sequence in this group.

When choosing the target region for investigation, is also important to consider the number of mtDNA sequences available in the taxon under study. The use of a region already characterized by different authors greatly facilitates the construction of databases and allows for the estimation of frequencies of mtDNA haplotypes and their geographic distributions. The mtDNA regions that have been regularly used in the forensic context are the noncoding control region (also known as the D-loop or AT-rich region) and the cytochrome b oxidase (CYTB), cytochrome c oxidase (COX 1, 2 and 3) and rRNA genes.

The control region is responsible for the precise binding of several nuclear-encoded proteins that regulate mtDNA replication and transcription $[2,31]$. In mammals, the nucleotide mutation rate is not uniformly distributed throughout the molecule [32], with a high number of rapidly evolving sites confined to the control region, which is usually organized into three different domains: a highly conserved central domain and two flanking adenine-rich segments with high intraspecific genetic variability, known as hypervariable regions I and II (HVRI and HVRII) [33]. These regions are of interest for the identification of closely related lineages because of their high degree of variation among individuals. Nevertheless, the control region in some invertebrate species is composed of more than $85 \%$ adenines and thymines and is often called the 'AT-rich region'. For instance, the A+T contents of the control regions of Caenorhabditis elegans (NC_001328.1) and Apis mellifera (NC_001566.1) are 93\% and $96 \%$, respectively. This aspect adds difficulty to the 


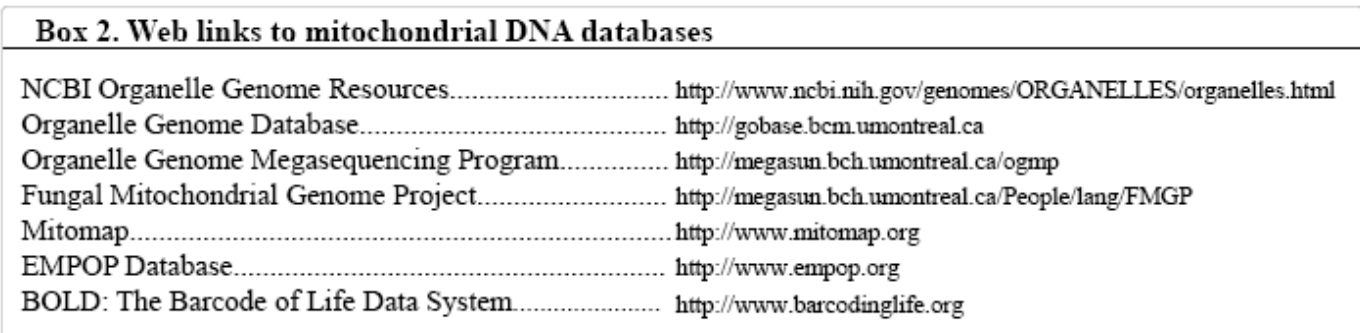

alignment of sequences and to the PCR amplification of homopolymeric stretches of As or Ts, which are very common in these regions.

A frequent feature of many control regions in a variety of vertebrate and invertebrate species is the presence of tandem repeats in both hypervariable regions, often predicted to fold into thermodynamically stable secondary structures [34]. The length of the repeated motif varies from just a few base pairs (e.g., 8 bp in Equus caballus) to over $700 \mathrm{bp}$ in the meadow grasshopper (Chorthippus parallelus) [34]. The rate of mutation to different size classes is high in many species and can be used to trace different lineages [35]. However, the use of these tandem repeats in forensics should be treated carefully since (a) the occurrence of length heteroplasmy due to the presence of mtDNAs with different number of repeated motifs in the same individual is common [36-38]; (b) repeat arrays of identical copy number may occur by convergence; (c) size-biased amplifications and null alleles may occur during PCR; and (d) detection and scoring of amplified arrays by conventional electrophoresis can insert significant errors (e.g., less abundant alleles may not be detected in poor gel staining) [34].

The effectiveness of hypervariable regions in the study of lineages with high phylogenetic divergence (e.g., different species) is offset by the mutational oversaturation of sites, with back- and parallel mutations confounding the identification. Therefore, forensic investigations aiming at the discrimination of divergent lineages have focused on less variable sites, such as those in protein-coding genes. In this regard, various gene regions have been employed for species-level analyses [21-23] (e.g., African elephant [39], shark [40], tiger [41] and blowfly [42]). The CYTB gene has been widely used, as it shows a high level of divergence between species but little intraspecies variation [21,43,44]. More recently, a 650-bp fragment of the COX1 gene has gained the designation 'DNA barcode' for the identification of animal species $[23,45]$ and was already validated for forensic use [46]. Nevertheless, some issues must be considered when employing any mtDNA gene for species identification procedures, namely, to verify that there is no overlap between intraspecific variation and interspecific divergence in the taxa under scrutiny and to guarantee the quality of DNA sequences deposited in the reference databases [47, 48]. In plants, the utility of these genes (and mitochondrial genomes at large) is limited by their low rate of sequence evolution, which does not provide species-level resolution [49]. For genetic identification purposes, the mitochondrial genome in plants has thus received far less attention than the chloroplast genome. Characteristics such as polyploidy, hybridization and asexual reproduction preclude the application of mtDNA markers in some species. Despite some limitations, mtDNA haplotyping has been successfully used to distinguish some varieties of cannabis (Cannabis sativa) [50] or to identify certain genera of grasses [51].

\section{BRIEF OVERVIEW OF LABORATORY PROCE- DURES FOR MTDNA TYPING}

A set of guidelines and recommendations for the analysis of mtDNA in forensic casework has been proposed in order to assure good laboratory practices and accurate interpretation of results $[11,13,20,52,53]$. The adoption of methods that have been previously accepted by the forensic community and regulatory boards is strongly advisable to facilitate court acceptance of results. All DNA profiling systems must be properly validated by the scientific community and by the laboratory conducting the analyses before implementation in forensic casework. The validation process depends on the procedure being verified but usually includes: characterization of the genetic marker, species specificity, reproducibility, precision, accuracy and sensitivity studies. The laboratory should participate in regular collaborative exercises and follow quality assurance practices to optimize procedures and adjust methods $[54,55]$. Further information on this topic can be found in specialized literature $[12,13,52,54$, $55]$ and at regular meetings organized by professional societies and/or working groups [56, 57].

The guiding principles for good laboratory practice in non-human mtDNA typing are similar to those proposed for dealing with human samples. The main concern should be to control and monitor contamination within the laboratory to guarantee high-quality results. The high copy number of mtDNA in most biological samples increases the risk of contamination. Some precautions against contamination include separate analyses of evidence items and reference samples, physical separation of pre- and post-PCR areas, UV light exposure of all materials and reagents used for pre-PCR operations, negative and positive controls in all reactions, replicate tests on separate occasions and examination of different tissues from the same individual (see references $[12,20,52$, 53] for details).

One of the first issues to be considered when planning the laboratory work is the technique to be used for DNA analysis. A large number of methods for typing mtDNA in humans have been described. The partial or complete sequencing of mtDNA is obviously the best way to retrieve more information from this genetic marker. But the choice of a particular technique is often a compromise that depends on the quality and quantity of the sample, availability of resources in the laboratory and time limitations. Some rapid low-resolution typing assays can be used to obtain partial information from mtDNA, which can be useful to screen samples for elimination from the investigation. The list of 
methods used for this purpose includes PCR-RFLP [58], microchip electrophoresis [59], sequence-specific oligonucleotide (SSO) dot blot assay, minisequencing [60] and pyrosequencing, among others (a complete list of techniques can be found in reference [12]). Nevertheless, the vast experience gathered by multiple inter-laboratory comparative exercises in human mtDNA typing have proven that PCRbased automated sequencing is a reliable method of forensic identification and should be used when possible [11-13, 20, 53].

Mitochondrial DNA can be extracted from the samples by standard techniques, but often the method has to be adapted to the type of biological evidence under examination (for instance, dry wood [61]). Nevertheless, the major concern should always be to preserve the DNA structure and to purify the sample of PCR inhibitors that may have been coextracted. One of the most effective extraction methods is the organic phenol-chloroform method followed by a subsequent purification step [62]. This method yields highmolecular-weight DNA but involves a number of procedural steps that increases the opportunity for error or laboratoryinduced contamination. A rapid method to extract highquality forensic samples is the Chelex extraction [63]. However, this method only separates the DNA from the cellular environment and does not extract the DNA from the residual components. The proteolytic component remains in solution and is known to inhibit PCR at high concentrations. It also produces single-stranded DNA, which is more susceptible to degradation and not effective for some typing methods (e.g., RFLP).

It is crucial to determine whether a sample will contain enough DNA for PCR amplification, since a sufficient amount of amplicon has to be produced in order to give a meaningful sequencing result. To estimate the quantity of mtDNA in the template sample is essential for most PCRbased assays that are optimized to work well on a narrow mtDNA concentration range. A total-DNA quantification method can facilitate estimations of the quantity of mtDNA only if the ratio between nuclear and mitochondrial DNA is well known. The direct determination of the number of mtDNA molecules in the sample can be performed by realtime PCR [64].

The choice of primers for PCR is also a very important step in laboratory procedures. When possible, the primers should be designed against evolutionarily conserved regions of the target sequence in order to avoid mutations in the primer-binding region that would reduce its affinity to the primer. If information from several individuals of the same species is available, a previous alignment of all mtDNA sequences is recommended in order to identify polymorphisms that could compromise the binding of primers during PCR. For instance, a single mismatch at the 3' end of a primer sequence leads to null amplification in certain PCR conditions. This step is even more problematic when primers are designed using sequences from other species or populations. It is also advisable to check all primers using a sequence alignment tool such as Basic Local Alignment Search Tool (BLAST; http://blast.ncbi.nlm.nih.gov/Blast.cgi) in order to test the theoretical specificity of primers. This kind of test is most crucial in species-identification applications. Nevertheless, the lack of match between designed primers and non- target species does not necessarily imply that primers are specific, since many species are not represented in databases.

A forensic laboratory often has to deal with highly degraded samples, where the DNA molecules could be randomly broken into smaller pieces by the action of nucleases or chemical reagents. Thus, the probability of having a successful PCR amplification usually decreases with the increase in the size of the target DNA region to amplify. A strategy used to retrieve information from degraded templates is to design PCRs for short amplifications. This approach has been used to amplify small portions (less than $300 \mathrm{bp}$ ) of human mtDNA [65, 66]. Several computer programs can help in the design of PCR primers for a desired amplicon size (e.g., Primer3; http://primer3.sourceforge.net/). The simultaneous amplification of two or more DNA regions in the same reaction (multiplex PCR) is another good example of a strategy to analyse low-quantity DNA samples. Simultaneously amplified fragments can then be used for independent sequencing or as a template for different DNA typing methods. Examples of multiplex PCR approaches can be found in references [65,67-69]. Potential primer-primer interactions should be avoided and can be detected by a few software programs (e.g., AutoDimer [70]).

The conditions of the PCR amplification depend on multiple factors, including the type of polymerase being used or the characteristics of the template DNA available for reaction. For instance, by increasing the number of cycles in the PCR, highly degraded or low-copy-number (less than $200 \mathrm{pg}$ template DNA) samples sometimes have a better chance of being amplified. Performing low-copy-number (LCN) typing includes adaptations of standard methodologies in order to increase the sensitivity of detection by means of a number of modifications to the standard methods. This approach and its validity in forensic science have recently been discussed [71] for the particular case of short tandem repeat (STR) analysis, but some of the issues presented therein are common to mtDNA analysis as well. Reducing the annealing temperature by a few degrees can facilitate the binding of primers that are not entirely complementary to the template DNA, as often occurs when primers are designed using the sequences of other species. However, any deviation from optimal PCR conditions to raise the sensitivity of the reaction also increases the chance of contamination and non-specific amplifications. Thus, a great deal of care is always required, particularly due to the abundance of mtDNA in most biological tissues.

\section{INTERPRETATION OF MTDNA DATA IN NON- HUMAN FORENSICS}

The main goal of using mtDNA in forensic tests is to help establish the source of a biological sample by means of lineage identification. It should be always clear that mtDNA does not have the power of discrimination possessed by STR systems for individual identification. All members of a matriline (or patriline, in some species) usually share the same mtDNA haplotype. A definitive individual identification using mtDNA is only possible in the rare event that the population of potential sources is closed and completely characterized and the haplotype from the questioned sample is not shared by different individuals. In most populations, the relative significance of a match has to be properly 
assessed using population databases. The assignment of an individual to a particular category (species, population, breed, etc.) is only possible if that group presents a unique set of mitochondrial lineages [22, 24, 47, 48]. This feature has to be demonstrated prior to the investigation by analysing a large number of individuals from the target and related groups. Dogs represent the most commonly analysed nonhuman source of forensic evidence, and a number of studies have focused the utility of mtDNA polymorphisms for the purpose of individual identification [72- 76].

A number of interpretation guidelines have been proposed, mainly for human identification purposes [11-13, 20, $52,53]$. Briefly, the procedure involves a comparison between the sequences obtained from known and evidence samples. To report the results, a reference sequence should be used, and only sites that differ from the reference should be scored. It is highly recommended to use the electropherograms (the graphical output of electrophoresis devices in sequencing analysis) directly and robust alignment algorithms, such as Muscle [77] (http://www.drive5.com/ muscle), Clustal [78] (http://www.clustal.org) or MAFFT [79] (http://align.bmr.kyushu-u.ac.jp/mafft/software), to compare the sequences. It is desirable to search for errors in alignments by hand and verify that all positions are correctly aligned, considering that most ambiguities arise with indel positions. Ideally, these tasks should be performed by more than one technician independently. To facilitate the process of sequence comparison, several software packages can be used for the alignment and the identification of differences: Geneious (http://www.geneious.com), Chromas (http://www. technelysium.com.au/chromas.html), BioEdit (http://www. mbio.ncsu.edu/BioEdit/bioedit.html), Sequencher (http:// www.sequencher.com) and several others. A list of recommendations to help clarify inconsistencies in alignments and a few examples can be found in reference [11].

The direct visual inspection of the sequencing peaks can facilitate the detection of heteroplasmy or sequencing artefacts, which may not be recognized by analysis of the text output file alone. The sample should be re-extracted and/or re-sequenced if the overall quality of the electropherogram is not sufficient for interpretation purposes. Moreover, it is highly recommended to sequence both strands of the amplicon in order to reduce ambiguities in sequence determination and also to carefully scrutinize all sequences for low levels of heteroplasmy. The routine sequencing of both DNA strands should be considered standard to ensure accurate results. The amplification strategy is also important for the detection of heteroplasmy [80]. If two bases are visible at the same site of both strands of a sequence, clearly above the background 'noise', the position can be interpreted as heteroplasmic (Fig. 4). Although it can complicate the interpretation of the results, heteroplasmy can actually increase the strength of evidence in some cases: similar heteroplasmic variation in two different samples is a rare event, particularly because heteroplasmic lineages become homoplasmic in just a few generations due to the rapid sorting of mtDNA molecules in germline cells [81]. In any case, good estimation of the heteroplasmy rates at that site is necessary to draw any definitive conclusion.

A problem often observed with conventional DNA sequencing approaches occurs in long homopolymeric stretches in the form of slippage of the DNA polymerases during replication. Enzyme slippage during PCR or sequencing reactions generates a mixture of DNA sequences with different lengths (for instance, a pool of mtDNAs with 16, 17 and 18 adenines in a particular A-stretch) that will cause an overlap or wave-like pattern in all peaks following the repetitive tract in the electropherogram (Fig. 5). A possible strategy to overcome this limitation is to design an alternative primer closer to the homopolymeric region, since the enzyme usually works better when extending smaller fragments. The sequencing of both strands and the use of internal primers to avoid homopolymeric tracts or tandem repeats is often necessary in order to obtain a double reading of each DNA fragment under study. However, it should be noted that a mixture of mtDNA molecules with different lengths can already be present in the sample if it originated from a heteroplasmic individual [82]. It has been shown that polymerase slippage during mtDNA replication in vivo can cause length heteroplasmy. In practice, length heteroplasmy in tandem repeats or mononucleotide stretches is much more frequently detected than sequence heteroplasmy [13, 34, 36$38,53,83]$.

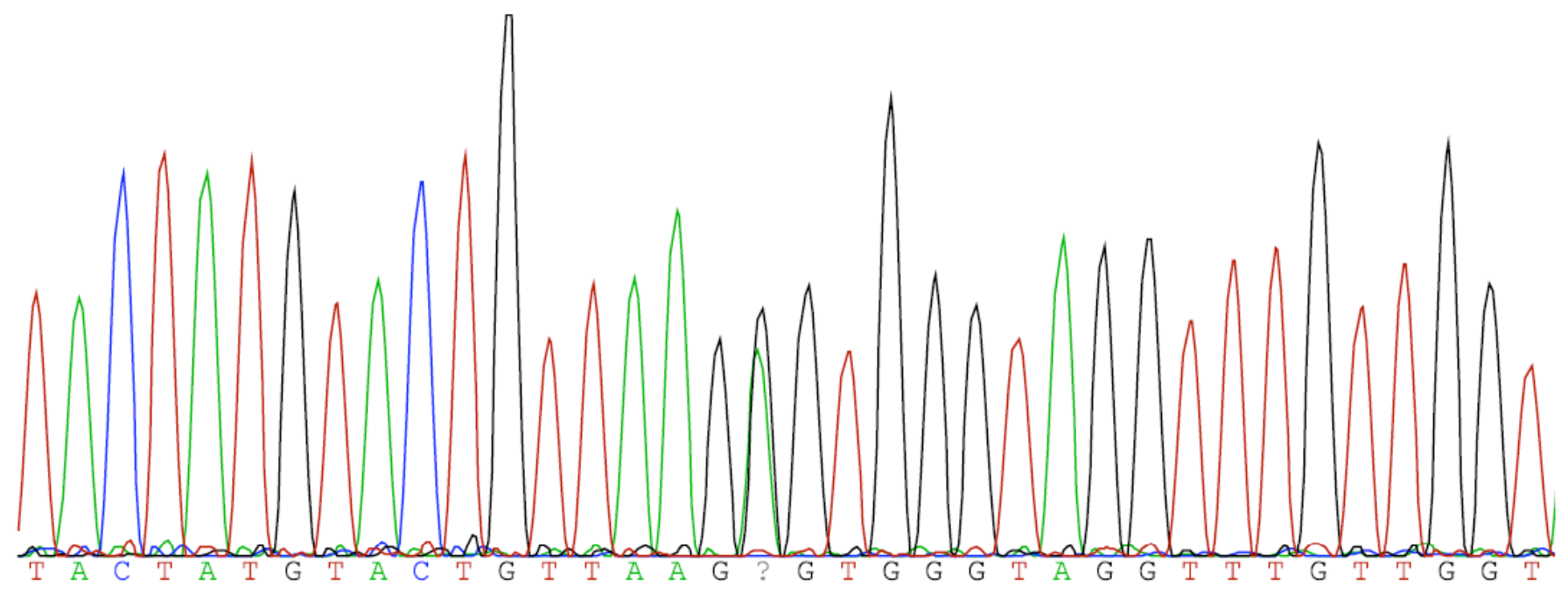

Fig. (4). An example of a point heteroplasmy in mitochondrial DNA. 


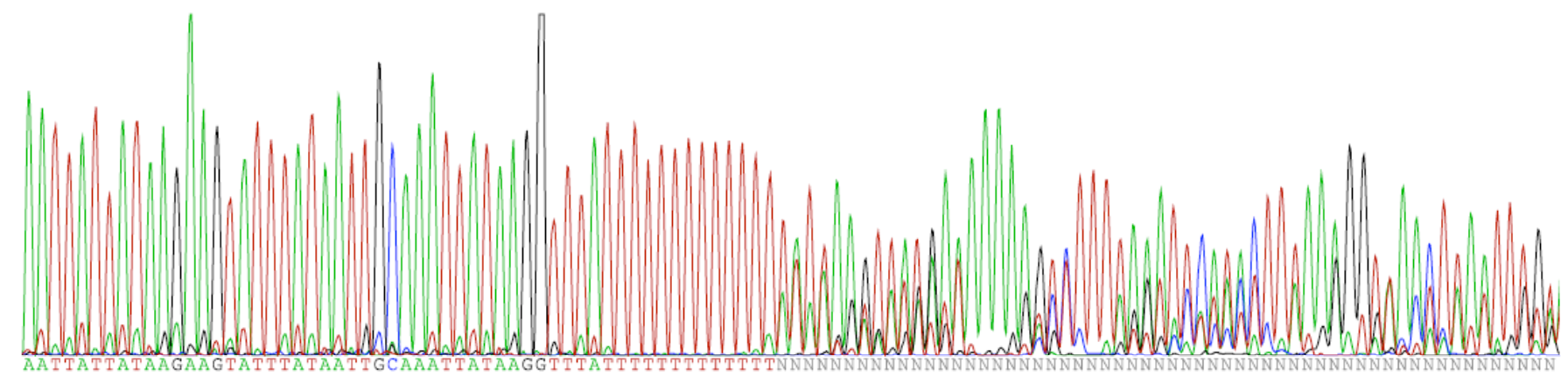

Fig. (5). Example of sequence slippage caused by a mononucleotide T-stretch. Two or more length variants appear as a mixed signal in the trace downstream of the homopolymer.

The existence of nuclear mitochondrial insertions (NUMTs) should also be taken into account when performing a PCR amplification of mtDNA from total genomic DNA, and much caution should be exerted when using mitochondrial sequences for identification purposes. These insertions result from the intracellular transfer of genetic material from the mitochondrion to the nucleus and can be very similar to mtDNA counterparts $[84,85]$. A large number of eukaryotic species (if not all) are known to have NUMTs (e.g., yeast, locust, sea urchin, human, maize and rat). It has been proposed that NUMTs can be inadvertently amplified in addition to or even instead of the target mtDNA. However, it has recently been shown that the risk of amplifying a NUMT rather than endogenous mtDNA is very low with conventional techniques and mtDNA-specific primers [86]. The high copy number of mtDNA in relation to any nuclear pseudogene works as a warranty against NUMT-derived contaminations. Nevertheless, current practices do not preclude inadvertent amplification of NUMTs, particularly if degenerate primers are designed from homologous sequences in relates species. The amplification of such nuclear insertions should be considered if (a) more than one amplified product is detected on an electrophoretic gel; (b) more than the expected number of ambiguities due to indels, frameshifts or unexpected stop codons are identified; and (c) the sequences are clearly different from those expected based on comparative genome analyses. A list of some measures to avoid the undesired amplification of NUMTs can be found at http://www.pseudogene.net.

The weight of the evidence based on mtDNA analysis must always take into account the peculiarities of this genome (heteroplasmy, high copy number, diverse mutational rates, etc.) and the limitations of the technology used. An example of a system of reporting results was proposed by the Scientific Working Group on DNA Analysis Methods (SWGDAM) for human mtDNA [87]. Three categories were defined: exclusion, inconclusive and cannot exclude (or failure to exclude). An 'exclusion' must be reported when the sequences are unequivocally different and present several nucleotide differences (if the sites are heteroplasmic, the interpretation could be different [11-13]). In this case, the samples can be excluded as originating from the same individual or maternal lineage (or paternal, in some species). An 'inconclusive' result must describe a situation in which two samples contain a small number of differences (one nucleotide difference, according to the SWGDAM for human
mtDNA) that occur in dubious positions, which can be interpreted as a result of somatic heteroplasmy or mother-to-son mutation. In this case, the strength of the evidence is less than that of a complete match, but it is not possible to report an exclusion. It is recommended to analyze additional samples from the same individual and to sequence other mtDNA regions in order to resolve the interpretation. The strength of the evidence also depends on the mutability of polymorphic bases, which should be estimated in phylogenetic and pedigree studies [88]. A single difference in a highly conserved site could indicate a lower probability of match than a difference in a hypervariable site. Nevertheless, a conservative approach is preferable in these cases since mutational hotspots can vary with the background sequence. For example, certain sequences are more prone to secondary structures $[89,90]$ that can affect the mutation rate. Therefore, the minimal number of differences to definitively exclude the questioned sample depends on the mtDNA region under analysis, mainly its length and mutability. It is obvious that the probability of finding differences in the same lineage or individual increases in species with very high rates of mtDNA sequence evolution. A good description of rates of mtDNA nucleotide-substitution mutations for several eukaryotic species can be found in the supporting online material of reference [25]. It has also been shown that mutations in human mtDNA are more likely to be found between different human tissues (e.g., hair and blood) than between two samples of the same tissue taken from the same individual [53]. The high energy demands of certain tissues and their mitotic or post-mitotic nature may also influence the expected distribution of mutations. It remains to be demonstrated if the same is true in other taxonomic groups.

Ultimately, if the mtDNA sequences from evidentiary and reference samples are equal, the samples cannot be excluded as originating from the same source. In a 'failure to exclude' situation, it is desirable to perform a statistical estimate of the significance of the match for reporting purposes. A simple way of doing this is to count the number of times a particular haplotype is observed in a population database (an approach known as a 'counting method') [11, 13, 53]. Thus, a reliable database is crucial for an accurate random match frequency estimate [88, 91-95]. It should include a large number of unrelated individuals that are potential contributors of evidence, preferentially from the full distribution range of the population, including isolated groups. The estimations should consider the possible existence of sub- 


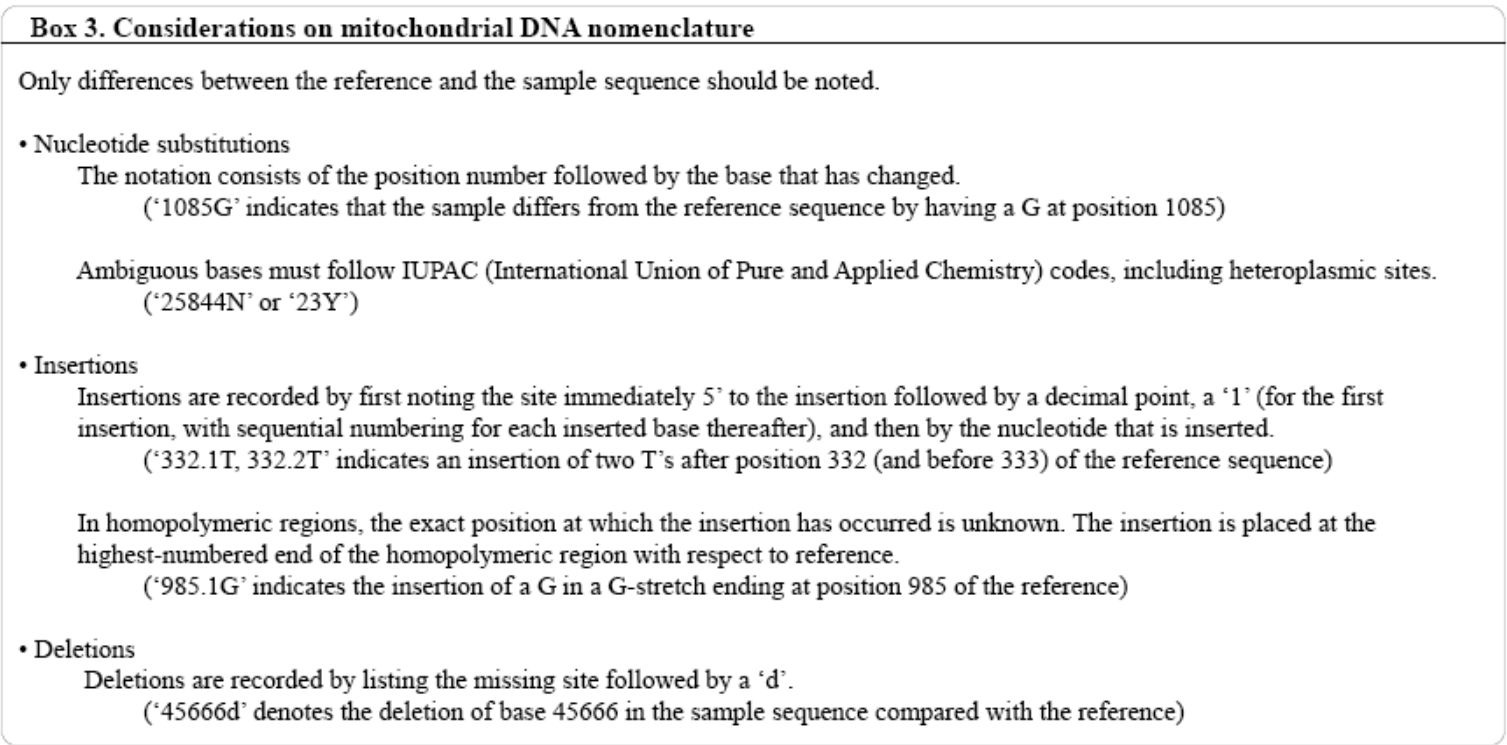

structure in the population and a correction for sampling errors (such as a confidence interval or a bootstrap correction). The nomenclature used to describe the profiles has to follow standardized rules accepted by the forensic community $[11,13,20,53]$. This problem has already been addressed for the mtDNA control region of the domestic dog [96]. Box 3 describes some basic rules used in mtDNA nomenclature.

In the last few years a large amount of flawed mtDNA data has been used for forensic and population genetics studies [88, 91-95]. The most common types of errors are base shifts, reference bias, phantom mutations, base misscoring and artificial recombination [94, 95]. Erroneous data can result from laboratory problems (e.g., contamination, sample mix-up, artefact sequencing), misalignment with or incorrect reference sequence, basecall misinterpretation, nomenclature violation and errors in the manual transcription of the raw sequence information to a final data table [93]. This can easily lead to underestimation or overestimation of the number of times an evidentiary sequence is found in a population database, and therefore matching probabilities may be inexactly calculated. A good way to prevent most mistakes and to evaluate the accuracy of databases is to consider the mtDNA phylogeny of the group under study. The guidelines proposed in references [88, 91,94] serve as a good starting point for avoiding different types of errors. An example of a mtDNA database that uses a variety of qualitycontrol analyses to check for sequence errors in submitted mtDNA sequences can be found at http://www.empop.org [97].

Finally, all conclusions of the forensic investigation should be reported in a concise way and include the calculations carried out to estimate the value of the mtDNA evidence. It is highly advisable to adopt statistical methods that have been previously accepted by the forensic community and the court system [98]. The final result should reflect a deep understanding on the advantages and limitations of mtDNA as a forensic tool and guarantee that reliable results have been obtained by following good laboratorial and analytic practices.

\section{ACKNOWLEDGEMENTS}

This work was supported by a research grant to FP (SFRH/BPD/44637/2008) and BA (SFRH/BDE/15581/2006) from Fundação para a Ciência e a Tecnologia. IPATIMUP is an Associate Laboratory of the Portuguese Ministry of Science, Technology and Higher Education and is partially supported by FCT, the Portuguese Foundation for Science and Technology. Authors' web site: http://www.portugene. $\mathrm{com} /$.

\section{REFERENCE}

[1] Gray, M.W.; Burger, G.; Lang, B.F. Mitochondrial evolution. Science, 1999, 283(5407), 1476-1481.

[2] Scheffler, I. Mitochondria; Second ed.; Jonh Wiley \& Sons, Inc: Hoboken, New Jersey, 2008.

[3] Smeitink, J.; van den Heuvel, L.; DiMauro, S. The genetics and pathology of oxidative phosphorylation. Nature Rev. Genet., 2001, 2(5), 342-352.

[4] Burger, G.; Gray, M.W.; Lang, B.F. Mitochondrial genomes: anything goes. Trends Genet., 2003, 19(12), 709-716.

[5] Shao, R.F.; Kirkness, E.F.; Barker, S.C. The single mitochondrial chromosome typical of animals has evolved into 18 minichromosomes in the human body louse, Pediculus humanus. Genome Res., 2009, 19(5), 904-912.

[6] Goremykin, V.V.; Salamini, F.; Velasco, R.; Viola, R. Mitochondrial DNA of Vitis vinifera and the issue of rampant horizontal gene transfer. Mol. Biol. Evol., 2009, 26(1), 99-110.

[7] Lynch, M. The Origins of Genome Architecture; Sinauer Associates Inc: 2007.

[8] Chinnery, P.F.; Samuels, D.C. Relaxed replication of mtDNA: A model with implications for the expression of disease. Am. J. Hum. Genet., 1999, 64(4), 1158-1165.

[9] Birky, C.W. The inheritance of genes in mitochondria and chloroplasts: Laws, mechanisms, and models. Ann. Rev. Genet., 2001, 35, 125-148.

[10] Wonnapinij, P.; Chinnery, P.F.; Samuels, D.C. The distribution of mitochondrial DNA heteroplasmy due to random genetic drift. Am. J. Hum. Genet., 2008, 83(5), 582-593.

[11] Budowle, B.; Allard, M.W.; Wilson, M.R.; Chakraborty, R. Forensics and mitochondrial DNA: Applications, debates, and foundations. Annu. Rev. Genomics Hum. Genet.,2003, 4, 119-141.

[12] Butler, J.M. Forensic DNA Typing; 2 ed.; Elsevier Academic Press: London, 2005.

[13] Holland, M.; Parsons, T.J. Mitochondrial DNA sequence analysis validation and use for forensic casework. Forensic Sci. Rev., 1999, 11(1), 21-50. 
[14] Breton, S.; Beaupre, H.D.; Stewart, D.T.; Hoeh, W.R.; Blier, P.U. The unusual system of doubly uniparental inheritance of mtDNA: isn't one enough? Trends Genet., 2007, 23(9), 465-474.

[15] Schwartz, M.; Vissing, J. Paternal inheritance of mtDNA in a patient with mitochondrial myopathy. Eur. J. Hum. Genet., 2002, 10, 239.

[16] Awadalla, P.; Eyre-Walker, A.; Smith, J.M. Linkage disequilibrium and recombination in hominid mitochondrial DNA. Science, 1999, 286(5449), 2524-2525.

[17] Macaulay, V.; Richards, M.; Sykes, B. Mitochondrial DNA recombination - no need to panic. Proc. R. Soc. Lond. Ser. B-Biol. Sci., 1999, 266(1433), 2037-2039.

[18] Chinnery, P.F.; Thorburn, D.R.; Samuels, D.C.; White, S.L.; Dahl, H.H.; Turnbull, D.M.; Lightowlers, R.N.; Howell, N. The inheritance of mitochondrial DNA heteroplasmy: random drift, selection or both? Trends Genet., 2000, 16(11), 500-505.

[19] Cree, L.M.; Samuels, D.C.; Lopes, S.C.D.S.; Rajasimha, H.K.; Wonnapinij, P.; Mann, J.R.; Dahl, H.H.M.; Chinnery, P.F. A reduction of mitochondrial DNA molecules during embryogenesis explains the rapid segregation of genotypes. Nat. Genet., 2008, 40(2), 249-254.

[20] Tully, G.; Bar, W.; Brinkmann, B.; Carracedo, A.; Gill, P.; Morling, N.; Parson, W.; Schneider, P. Considerations by the European DNA profiling (EDNAP) group on the working practices, nomenclature and interpretation of mitochondrial DNA profiles. Forensic Sci. Int., 2001, 124(1), 83-91.

[21] Parson, W.; Pegoraro, K.; Niederstatter, H.; Foger, M.; Steinlechner, M. Species identification by means of the cytochrome $b$ gene. Int. J. Legal Med., 2000, 114(1-2), 23-28.

[22] Pereira, F.; Carneiro, J.; Amorim, A. Identification of species with DNA-based technology: current progress and challenges. Recent Pat. DNA Gene Seq., 2008, 2, 187-200.

[23] Ratnasingham, S.; Hebert, P.D.N. BOLD: The Barcode of Life Data System (www.barcodinglife.org). Mol. Ecol. Notes, 2007, 7(3), 355-364.

[24] Wells, J.D.; Stevens, J.R. Application of DNA-based methods in forensic entomology. Annu. Rev. Entomol., 2008, 53, 103-120.

[25] Lynch, M.; Koskella, B.; Schaack, S. Mutation pressure and the evolution of organelle genomic architecture. Science, 2006, 311(5768), 1727-1730.

[26] Knoop, V. The mitochondrial DNA of land plants: peculiarities in phylogenetic perspective. Curr. Genet., 2004, 46(3), 123-139.

[27] Kubo, T.; Newton, K.J. Angiosperm mitochondrial genomes and mutations. Mitochondrion, 2008, 8(1), 5-14.

[28] Fauron, C.; Casper, M.; Gao, Y.; Moore, B. The maize mitochondrial genome - dynamic, yet functional. Trends Genet., 1995, 11(6), 228-235.

[29] Coulthart, M.B.; Huh, G.S.; Gray, M.W. Physical organization of the 18S-ribosomal and 5S-ribosomal RNA genes in the mitochondrial genome of rye (Secale-Cereale L). Curr. Genet., 1990, 17(4), 339-346.

[30] Beagley, C.T.; Okimoto, R.; Wolstenholme, D.R. The mitochondrial genome of the sea anemone Metridium senile (Cnidaria): Introns, a paucity of tRNA genes, and a near-standard genetic code. Genetics, 1998, 148(3), 1091-1108.

[31] Falkenberg, M.; Larsson, N.G.; Gustafsson, C.M. DNA replication and transcription in mammalian mitochondria. Аnпи. Rev. Biochem., 2007, 76, 679-699.

[32] Galtier, N.; Enard, D.; Radondy, Y.; Bazin, E.; Belkhir, K. Mutation hot spots in mammalian mitochondrial DNA. Genome Res., 2006, 16(2), 215-222.

[33] Sbisa, E.; Tanzariello, F.; Reyes, A.; Pesole, G.; Saccone, C. Mammalian mitochondrial D-loop region structural analysis: identification of new conserved sequences and their functional and evolutionary implications. Gene, 1997, 205(1-2), 125-140.

[34] Lunt, D.H.; Whipple, L.E.; Hyman, B.C. Mitochondrial DNA variable number tandem repeats (VNTRs): utility and problems in molecular ecology. Mol. Ecol., 1998, 7(11), 1441-1455.

[35] Savolainen, P.; Arvestad, L.; Lundeberg, J. A novel method for forensic DNA investigations: Repeat-type sequence analysis of tandemly repeated mtDNA in domestic dogs. J. Forensic Sci., 2000, 45(5), 990-999.

[36] Buroker, N.E.; Brown, J.R.; Gilbert, T.A.; O'Hara, P.J.; Beckenbach, A.T.; Thomas, W.K.; Smith, M.J. Length heteroplasmy of sturgeon mitochondrial DNA: an illegitimate elongation model. Genetics, 1990, 124(1), 157-163.

[37] Savolainen, P.; Arvestad, L.; Lundeberg, J. mtDNA tandem repeats in domestic dogs and wolves: mutation mechanism studied by analysis of the sequence of imperfect repeats. Mol. Biol. Evol., 2000, 17(4), 474-488.

[38] Wilkinson, G.S.; Chapman, A.M. Length and sequence variation in evening bat D-loop mtDNA. Genetics, 1991, 128(3), 607-617.

[39] Comstock, K.E.; Ostrander, E.A.; Wasser, S.K. Amplifying nuclear and mitochondrial DNA from African elephant ivory: A tool for monitoring the ivory trade. Conserv. Biol., 2003, 17(6), 1840-1843.

[40] Hoelzel, A.R. Shark fishing in fin soup. Conserv. Genet., 2001, 2, 69-72.

[41] Wan, Q. H.; Fang, S.G. Application of species-specific polymerase chain reaction in the forensic identification of tiger species. Forensic Sci. Int., 2003, 131(1), 75-78.

[42] Wallman, J.F.; Donnellan, S.C. The utility of mitochondrial DNA sequences for the identification of forensically important blowflies (Diptera: Calliphoridae) in southeastern Australia. Forensic Sci. Int., 2001, 120(1-2), 60-67.

[43] Branicki, W.; Kupiec, T.; Pawlowski, R. Validation of cytochrome $\mathrm{b}$ sequence analysis as a method of species identification. J Forensic Sci., 2003, 48(1), 83-87.

[44] Hsieh, H.M.; Chiang, H.L.; Tsai, L.C.; Lai, S.Y.; Huang, N.E.; Linacre, A.; Lee, J.C. Cytochrome b gene for species identification of the conservation animals. Forensic Sci. Int., 2001, 122(1), 7-18.

[45] Hebert, P.D.; Cywinska, A.; Ball, S.L.; Dewaard, J.R. Biological identifications through DNA barcodes. Proc. Biol. Sci., 2003, 270(1512), 313-321.

[46] Dawnay, N.; Ogden, R.; McEwing, R.; Carvalho, G.R.; Thorpe, R.S. Validation of the barcoding gene COI for use in forensic genetic species identification. Forensic Sci. Int., 2007, 173(1), 1-6.

[47] Meyer, C.P.; Paulay, G. DNA barcoding: error rates based on comprehensive sampling. PLoS Biol., 2005, 3(12), e422.

[48] Moritz, C.; Cicero, C. DNA barcoding: promise and pitfalls. PLoS Biol., 2004, 2(10), e354.

[49] Fazekas, A.J.; Kesanakurti, P.R.; Burgess, K.S.; Percy, D.M.; Graham, S.W.; Barrett, S.C.H.; Newmaster, S.G.; Hajibabaei, M.; Husband, B.C. Are plant species inherently harder to discriminate than animal species using DNA barcoding markers? Mol. Ecol. Resour., 2009, 9, 130-139.

[50] Gilmore, S.; Peakall, R.; Robertson, J. Organelle DNA haplotypes reflect crop-use characteristics and geographic origins of Cannabis sativa. Forensic Sci. Int., 2007, 172(2-3), 179-190.

[51] Ward, J.; Peakall, R.; Gilmore, S.R.; Robertson, J. A molecular identification system for grasses: a novel technology for forensic botany. Forensic Sci. Int., 2005, 152(2-3), 121-131.

[52] Budowle, B.; Garofano, P.; Hellman, A.; Ketchum, M.; Kanthaswamy, S.; Parson, W.; van Haeringen, W.; Fain, S.; Broad, T. Recommendations for animal DNA forensic and identity testing. Int. J. Legal Med., 2005, 119(5), 295-302.

[53] Carracedo, A.; Bar, W.; Lincoln, P.; Mayr, W.; Morling, N.; Olaisen, B.; Schneider, P.; Budowle, B.; Brinkmann, B.; Gill, P.; Holland, M.; Tully, G.; Wilson, M. DNA commission of the international society for forensic genetics: guidelines for mitochondrial DNA typing. Forensic Sci. Int., 2000, 110(2), 79-85.

[54] Prieto, L.; Alonso, A.; Alves, C.; Crespillo, M.; Montesino, M.; Picornell, A.; Brehm, A.; Ramirez, J. L.; Whittle, M.R.; Anjos, M.J.; Boschi, I.; Buj, J.; Cerezo, M.; Cardoso, S.; Cicarelli, R.; Comas, D.; Corach, D.; Doutremepuich, C.; Espinheira, R.M.; Fernandez-Fernandez, I.; Filippini, S.; Garcia-Hirschfeld, J.; Gonzalez, A.; Heinrichs, B.; Hernandez, A.; Leite, F.P.N.; Lizarazo, R.P.; Lopez-Parra, A.M.; Lopez-Soto, M.; Lorente, J.A.; Mechoso, B.; Navarro, I.; Pagano, S.; Pestano, J.J.; Puente, J.; Raimondi, E.; Rodriguez-Quesada, A.; Terra-Pinheiro, M.F.; Vidal-Rioja, L.; Vullo, C.; Salas, A. 2006 GEP-ISFG collaborative exercise on mtDNA: reflections about interpretation, artefacts, and DNA mixtures. Forensic Sci. Int.: Genet., 2008, 2(2), 126-133.

[55] van Asch, B.; Albarran, C.; Alonso, A.; Angulo, R.; Alves, C.; Betancor, E.; Catanesi, C.I.; Corach, D.; Crespillo, M.; Doutremepuich, C.; Estonba, A.; Fernandes, A.T.; Fernandez, E.; Garcia, A.M.; Garcia, M.A.; Gilardi, P.; Goncalves, R.; Hernandez, A.; 
Lima, G.; Nascimento, E.; de Pancorbo, M.M.; Parra, D.; Pinheiro, M.d.F.; Prat, E.; Puente, J.; Ramirez, J.L.; Rendo, F.; Rey, I.; Di Rocco, F.; Rodriguez, A.; Sala, A.; Salla, J.; Sanchez, J.J.; Sola, D.; Silva, S.; Pestano Brito, J.J.; Amorim, A. Forensic analysis of dog (Canis lupus familiaris) mitochondrial DNA sequences: An inter-laboratory study of the GEP-ISFG working group. Forensic Sci. Int. Genet., 2009, 4(1), 49-54.

[56] Non-Human Forensic Genetics Commission of the Spanish and Portuguese Working Group of the International Society for Forensic Genetics (http://www.gep-isfg.org).

[57] International Society of Animal Genetics (www.isag.org.uk).

[58] Pushnova, E.A.; Akhmedova, S.N.; Shevtsov, S.P.; Schwartz, E.I. A rapid and simple DNA-fingerprinting method using RLFP and SSCP analysis of the hypervariable noncoding region of human mitochondrial DNA. Hum. Mutat., 1994, 3(3), 292-296.

[59] Alonso, A.; Albarran, C.; Martin, P.; Garcia, P.; Capilla, J.; Garcia, O.; de la Rua, C.; Izaguirre, N.; Pereira, F.; Pereira, L.; Amorim, A.; Sancho, M. Usefulness of microchip electrophoresis for the analysis of mitochondrial DNA in forensic and ancient DNA studies. Electrophoresis, 2006, 27(24), 5101-5109.

[60] Vallone, P.M.; Just, R.S.; Coble, M.D.; Butler, J.M.; Parsons, T.J. A multiplex allele-specific primer extension assay for forensically informative SNPs distributed throughout the mitochondrial genome. Int. J. Legal Med., 2004, 118(3), 147-157.

[61] Deguilloux, M.F.; Pemonge, M.H.; Petit, R.J. Novel perspectives in wood certification and forensics: dry wood as a source of DNA. Proc. R. Soc. B-Biol. Sci., 2002, 269(1495), 1039-1046.

[62] Köchl, S.; Niederstätte, H.; Parson, W. DNA extraction and quantitation of forensic samples using the phenol-chloroform method and real-time PCR. Methods Mol. Biol., 2005, 297, 13-30.

[63] Walsh, P.S.; Metzger, D.A.; Higuchi, R. Chelex-100 as a medium for simple extraction of DNA for PCR-based yyping from forensic material. Biotechniques, 1991, 10(4), 506-513.

[64] Timken, M.D.; Swango, K.L.; Orrego, C.; Buoncristiani, M.R. A duplex real-time qPCR assay for the quantification of human nuclear and mitochondrial DNA in forensic samples: Implications for quantifying DNA in degraded samples. J. Forensic Sci., 2005, 50(5), 1044-1060.

[65] Alonso, A.; Albarran, C.; Martin, P.; Garcia, P.; Garcia, O.; de la Rua, C.; Alzualde, A.; Fernandez de Simon, L.; Sancho, M.; Fernandez Piqueras, J. Multiplex-PCR of short amplicons for mtDNA sequencing from ancient DNA. Int. Congress Ser., 2003, 1239, 585-588.

[66] Berger, C.; Parson, W. Mini-midi-mito: Adapting the amplification and sequencing strategy of mtDNA to the degradation state of crime scene samples. Forensic Sci. Int.: Genet., 2009, 3(3), 149153.

[67] Bataille, M.; Crainic, K.; Leterreux, M.; Durigon, M.; de Mazancourt, P. Multiplex amplification of mitochondrial DNA for human and species identification in forensic evaluation. Forensic Sci. Int., 1999, 99(3), 165-170.

[68] Eichmann, C.; Parson, W. 'Mitominis': multiplex PCR analysis of reduced size amplicons for compound sequence analysis of the entire mtDNA control region in highly degraded samples. Int. J. Legal Med., 2008, 122(5), 385-388.

[69] Krause, J.; Dear, P.H.; Pollack, J.L.; Slatkin, M.; Spriggs, H.; Barnes, I.; Lister, A.M.; Ebersberger, I.; Paabo, S.; Hofreiter, M. Multiplex amplification of the mammoth mitochondrial genome and the evolution of Elephantidae. Nature, 2006, 439(7077), 724727.

[70] Vallone, P.M.; Butler, J.M. AutoDimer: a screening tool for primer-dimer and hairpin structures. Biotechniques, 2004, 37(2), 226-231.

[71] Budowle, B.; Eisenberg, A.J.; van Daal, A. Validity of low copy number typing and applications to forensic science. Croat. Med. J., 2009, 50(3), 207-217.

[72] Angleby, H.; Savolainen, P. Forensic informativity of domestic dog mtDNA control region sequences. Forensic Sci. Int., 2005, 154(2-3), 99-110.

[73] Baute, D.T.; Satkoski, J.A.; Spear, T.F.; Smith, D.G.; Dayton, M.R.; Malladi, V.S.; Goyal, V.; Kou, A.; Kinaga, J.L.; Kanthaswamy, S. Analysis of forensic SNPs in the canine mtDNA HV1 mutational hotspot region. J. Forensic Sci., 2008, 53(6), 13251333.
[74] Eichmann, C.; Parson, W. Molecular characterization of the canine mitochondrial DNA control region for forensic applications 1. Int. J. Legal Med., 2007, 121(5), 411-416.

[75] Himmelberger, A.L.; Spear, T.F.; Satkoski, J.A.; George, D.A.; Garnica, W.T.; Malladi, V.S.; Smith, D.G.; Webb, K.M.; Allard, M.W.; Kanthaswamy, S. Forensic utility of the mitochondrial hypervariable region 1 of domestic dogs, in conjunction with breed and geographic information. J. Forensic Sci., 2008, 53(1), 81-89.

[76] Webb, K.M.; Allard, M.W. Mitochondrial genome DNA analysis of the domestic dog: Identifying informative SNPs outside of the control region. J. Forensic Sci., 2009, 54(2), 275-288.

[77] Edgar, R.C. MUSCLE: multiple sequence alignment with high accuracy and high throughput. Nucleic Acids Res., 2004, 32(5), 1792-1797.

[78] Larkin, M.A.; Blackshields, G.; Brown, N.P.; Chenna, R.; McGettigan, P.A.; McWilliam, H.; Valentin, F.; Wallace, I.M.; Wilm, A.; Lopez, R.; Thompson, J.D.; Gibson, T.J.; Higgins, D.G. Clustal W and clustal X version 2.0. Bioinformatics, 2007, 23(21), 2947-2948.

[79] Katoh, K.; Toh, H. Recent developments in the MAFFT multiple sequence alignment program. Brief. Bioinformatics, 2008, 9(4), 286-298.

[80] Brandstatter, A.; Parson, W. Mitochondrial DNA heteroplasmy or artefacts - a matter of the amplification strategy? Int. J. Legal Med., 2003, 117(3), 180-184.

[81] Avise, J.C.; Arnold, J.; Ball, R.M.; Bermingham, E.; Lamb, T.; Neigel, J.E.; Reeb, C.A.; Saunders, N.C. Intraspecific phylogeography - the mitochondrial-DNA bridge between populationgenetics and systematics. Ann. Rev. Ecol. Syst., 1987, 18, 489522 .

[82] Irwin, J.A.; Saunier, J.L.; Niederstatter, H.; Strouss, K.M.; Sturk, K.A.; Diegoli, T.M.; Brandstatter, A.; Parson, W.; Parsons, T.J. Investigation of heteroplasmy in the human mitochondrial DNA control region: A synthesis of observations from more than 5000 global population samples. J. Mol. Evol., 2009, 68(5), 516527.

[83] Salas, A.; Lareu, M.V.; Carracedo, A. Heteroplasmy in mtDNA and the weight of evidence in forensic mtDNA analysis: a case report. Int. J. Legal Med., 2001, 114(3), 186-190.

[84] Bensasson, D.; Zhang, D.X.; Hartl, D.L.; Hewitt, G.M. Mitochondrial pseudogenes: evolution's misplaced witnesses. Trends Ecol. Evol., 2001, 16(6), 314-321.

[85] Leister, D. Origin, evolution and genetic effects of nuclear insertions of organelle DNA. Trends Genet., 2005, 21(12), 655663.

[86] Goios, A.; Prieto, L.; Amorim, A.; Pereira, L. Specificity of mtDNA-directed PCR - influence of NUclear MTDNA insertion (NUMT) contamination in routine samples and techniques. Int. J. Legal Med., 2008, 122(4), 341-345.

[87] Scientific Working Group on DNA Analysis Methods (SWGDAM) Guidelines for mitochondrial DNA (mtDNA) nucleotide sequence interpretation. Forensic Sci. Commun., 2003, 5(2), online.

[88] Salas, A.; Bandelt, H.J.; Macaulay, V.; Richards, M.B. Phylogeographic investigations: The role of trees in forensic genetics. Forensic Sci. Int., 2007, 168(1), 1-13.

[89] Brown, G.G.; Gadaleta, G.; Pepe, G.; Saccone, C.; Sbisa, E. Structural conservation and variation in the D-loop-containing region of vertebrate mitochondrial DNA. J. Mol. Biol., 1986, 192(3), 503-511.

[90] Pereira, F.; Soares, P.; Carneiro, J.; Pereira, L.; Richards, M.B.; Samuels, D.C.; Amorim, A. Evidence for variable selective pressures at a large secondary structure of the human mitochondrial DNA control region. Mol. Biol. Evol., 2008, 25(12), 2759-2770.

[91] Bandelt, H.J.; Lahermo, P.; Richards, M.; Macaulay, V. Detecting errors in mtDNA data by phylogenetic analysis. Int. J. Legal Med., 2001, 115(2), 64-69.

[92] Bandelt, H.J.; Kivisild, T. Quality assessment of DNA sequence data: Autopsy of a mis-sequenced mtDNA population sample. Ann. Hum. Genet., 2006, 70, 314-326.

[93] Bandelt, H.J.; Kivisild, T.; Parik, J.; Villems, R.; Bravi, C.M.; Yao, Y.G.; Brandstatter, A.; Parson, W. Lab-Specific Mutation Processes. In: Human Mitochondrial DNA and the Evolution of Homo 
sapiens. Bandelt, H. J., Macaulay, V., Richards, M. B., Eds.; Springer-Verlag: Berlin Heidelberg, 2006, vol. 18.

[94] Salas, A.; Carracedo, A.; Macaulay, V.; Richards, M.; Bandelt, H.R. A practical guide to mitochondrial DNA error prevention in clinical, forensic, and population genetics. Biochem. Biophys. Res. Commun., 2005, 335(3), 891-899.

[95] Yao, Y.G.; Bravi, C.M.; Bandelt, H.J. A call for mtDNA data quality control in forensic science. Forensic Sci. Int., 2004, 141(1), $1-6$.
[96] Pereira, L.; van Asch, B.; Amorim, A. Standardisation of nomenclature for dog mtDNA D-loop: a prerequisite for launching a Canis familiaris database. Forensic Sci. Int., 2004, 141, 99-108.

[97] Parson, W.; Dur, A. EMPOP - A forensic mtDNA database. Forensic Sci. Int. Genet., 2007, 1(2), 88-92.

[98] Buckleton, J.; Walsh, S.W.; Harbison, S. Nonautosomal forensic markers. In Forensic DNA evidence interpretation, Buckleton, J., Triggs, C.M., Walsh, S.W., Eds.; CRS Press, USA: 2005; pp 299340 .

(C) Pereira et al.; Licensee Bentham Open.

This is an open access article licensed under the terms of the Creative Commons Attribution Non-Commercial License (http://creativecommons.org/licenses/by-nc/3.0/) which permits unrestricted, non-commercial use, distribution and reproduction in any medium, provided the work is properly cited. 